\title{
Multiple Kernel Learning with Maximum Inundation Extent from MODIS Imagery for Spatial Prediction of Flood Susceptibility
}

\section{Qiang $\mathrm{Hu}$}

Hohai University

\section{Yuelong Zhu}

Hohai University

Hexuan Hu ( $\square$ hexuan_hu@hhu.edu.cn )

Hohai University

\section{Zhuang Guan}

Hohai University

\section{Zeyu Qian}

Hohai University

\section{Aiming Yang}

Changjiang Water Resources Commission

\section{Research Article}

Keywords: Flood susceptibility, Multiple kernel learning, Support vector machine, Nonlinear combination, Maximum inundation extent

Posted Date: July 21 st, 2021

DOl: https://doi.org/10.21203/rs.3.rs-685721/v1

License: (c) (1) This work is licensed under a Creative Commons Attribution 4.0 International License.

Read Full License

Version of Record: A version of this preprint was published at Water Resources Management on November 8th, 2021. See the published version at https://doi.org/10.1007/s11269-021-03010-2. 


\title{
Multiple Kernel Learning with Maximum Inundation Extent from MODIS
}

\section{Imagery for Spatial Prediction of Flood Susceptibility}

Qiang $\mathrm{Hu}^{1}$, Yuelong Zhu ${ }^{1}$, Hexuan $\mathrm{Hu}^{1,2}$, Zhuang Guan ${ }^{1}$, Zeyu Qian ${ }^{1}$, Aiming Yang ${ }^{1,2,3}$

${ }^{1}$ College of Computer and Information, Hohai University, Nanjing, 211100, China

${ }^{2}$ Electric Engineering College, Tibet Agriculture \& Animal Husbandry University, Tibet, 860000, China

${ }^{3}$ Changjiang Survey, Planning, Design and Research Co., Ltd, Wuhan 430010, China

${ }^{4}$ Changjiang Spatial Information Technology Engineering Co., Ltd, Wuhan 430010, China

${ }^{5}$ Water Resources Information Perception and Big Data Engineering Research Center of Hubei Province, Wuhan 430010, China

Corresponding author at: Room 4309, Qinxue Building, No.8 Focheng West Road, Jiangning District, Nanjing, 211100, China.

E-mail address: hexuan_hu@hhu.edu.cn (H. Hu)

\begin{abstract}
:
This paper proposes a new technology of spatial prediction for flood susceptibility. Multiple kernel learning was used to build the flood susceptibility model and predict the flood inundation risk of the Sanhuajian Basin of the Yellow River. Based on the historical flow records of the Huayuankou Site and the MODIS remote sensing images of the study area, the maximum inundation range was extracted by the open water
\end{abstract}


likelihood index method, and the flooded and non-flooded sample sites were selected. Considering the availability of pertinent literatures and data, ten flood conditioning factors were defined as the sample characteristics. The model performance was evaluated in terms of accuracy, F1 score, and AUC. According to the results, multiple kernel learning significantly outperforms the support vector machine adopting single kernel, and NLMKL demonstrates the best comprehensive performance. The flood susceptibility map generated by MODIS remote sensing images and multiple kernel learning, therefore, can provide effective help for researchers and decision makers in flood management.

Keywords: Flood susceptibility; Multiple kernel learning; Support vector machine; Nonlinear combination; Maximum inundation extent

\section{Introduction}

Flood is one of the most destructive natural disasters. It inflicts massive losses all over the world on a yearly basis (Chen et al. 2020). From 1980 to 2016, flood accounted for 23\% of global economic loss (Mateo et al. 2017). Such losses can be mitigated or even averted by effective flood risk management methods.

Hydrodynamics-based flood inundation models, such as HEC-RAS (Pappenberger et al. 2005), Mike21 (Beilicci et al. 2016), Delft3D (Hoch et al. 2018), LISFLOOD-FP (Rajib et al. 2020), etc., can provide detailed and high-precision information on the spatial distribution of flood risks in a river basin so as to facilitate decision-making. These models, however, have some limitations in that they require high-standard data and intensive calculation (Teng et al. 2017).

Compared with flood risk maps generated by hydraulic models, the flood susceptibility map (FSM), which has been widely studied in recent years, exhibits 
superior cross-regional portability and convenience (Tehrany et al. 2013; Shafapour et al. 2019; Zhao et al. 2019; Tien et al. 2020; Yariyan et al. 2020). FSM adopts the geographic information system (GIS) in conjunction with machine learning to predict flood probability and evaluate the risk in the study area. Essential to the construction of an FSM machine learning model are flood conditioning factors (including altitude, slope, soil, rainfall, etc.) and historical records of flood inundation of the study area.

In the actual modelling process, however, insufficiency and poor availability of the historical data (labeled data) is a commonplace issue. Moreover, since the majority of studies only focus on a single flood outbreak in the study area, the findings are prone to have limitations. In view of this, we employed the open water likelihood index (OWL) method to extract the inundation range from MODIS remote sensing images in the study area (Huang et al. 2014), worked out the historical maximum inundation range by synthesizing multiple inundation ranges throughout a long time series, and used this as the base map for the sampling of the machine learning model.

In terms of machine learning algorithms, the support vector machine (SVM) has been proved to be suitable for FSM (Shafapour et al. 2019; Zhao et al. 2019), and the kernel function selection and parameter optimization are the key to performance. Studies in this field have mostly adopted SVM with a single kernel function, which has a relatively narrow variation space as well as limited generalization ability and robustness.

In recent years, multiple kernel learning (MKL) has become a hotspot in machine learning researches and been widely used in imaging (Gu et al. 2016), bioinformatics (Qi et al. 2020), and finance (Arratia et al. 2020). MKL learns through a combination of multiple kernel functions instead of one, and the kernel combination can be linear, nonlinear, or data dependent (Gönen et al. 2011). The MKL obtained by optimizing the 
combination of base kernels shows better generalization performance as it can eliminate the heterogeneous information in sample features and uneven distribution of data in high-dimensional feature space (Rakotomamonjy et al. 2008; Gönen et al. 2011, 2013; Zheng et al. 2017).

This research focuses on using different combinations of MKL to classify the flood susceptibility in the study area and draw the FSM. Central to MKL is the combination of multiple kernel functions and the learning method for the base kernel weight coefficient. EasyMKL, a scalable MKL algorithm (Aiolli et al. 2015), is adopted for the linear kernel function combination. Combining the basic kernels by solving a quadratic programming problem, it features a low memory consumption and a short computing time. For the nonlinear kernel combination, NLMKL (Cortes et al. 2009) is used, which can be integrated with SVM and transform the min-max problem into a simpler minimization problem. A momentum-based gradient descent algorithm is proposed to solve the optimal weight of each kernel matrix. We applied the two MKL algorithms to FSM research, investigating the area between Sanmenxia and Huayuankou in the Yellow River Basin of China. According to the results, multiple kernel learning significantly outperforms the support vector machine adopting single kernel or other baseline methods, and NLMKL has the best comprehensive performance.

The remaining part of this paper is organized as follows. The section 2 is an introduction to multiple kernel learning. The section 3 introduces the method of extracting the maximum inundation range from MODIS Imagery. The section 4 exhibits the data of the study area. The section 5 shows the experimental results and discussions with regard to the comparison between the several algorithms. Finally, the conclusion is drawn in the section 6 . 


\section{Proposed Method}

Support vector machines often use a single kernel function to measure the similarity between different instances. The selection of the kernel function and its parameters determines the structure of feature space as well as the classification effect (Damasevicius et al. 2010). However, a single kernel function cannot fully describe data similarity. MKL, in contrast, combines multiple kernel functions and thus often outperforms single kernel SVM. The kernel combination of MKL can be linear (weighted addition of multiple kernels), nonlinear (product or power of multiple kernels) or data dependent (kernel combination in relation to sample characteristics). By finding the best kernel combination parameters, MKL combines several base kernels and makes use of their respective advantages to obtain a kernel function with stronger learning and generalization abilities. Common methods of determining the kernel combination parameters include the fixed rule-based method, heuristic method, and optimization method (Gönen et al. 2011).

\subsection{EasyMKL}

EasyMKL (Aiolli et al. 2015), a highly scalable MKL algorithm, can effectively handle hundreds of thousands of kernels. Compared with other MKL algorithms (e.g. Simple MKL, generalized MKL, etc.), it shows better performance and maintains good robustness even in the presence of data noise. This algorithm has a linear combination of the base kernels in the following form:

$$
\boldsymbol{K}=\sum_{r=1}^{R} \eta_{r} \boldsymbol{K}_{r}, \eta_{r} \geq 0
$$

Where $\boldsymbol{K}_{r}$ is the base kernel function, $\mathrm{R}$ is the number of base kernels, and $\eta_{r}$, the weight coefficient of each base kernel. EasyMKL obtains the best combination parameters by solving a min-max problem about the variables $\boldsymbol{\gamma}$ and $\boldsymbol{\eta}$ : 


$$
\max _{\eta:\|\eta\|=1} \min _{\gamma \in \Gamma}(1-\lambda) \boldsymbol{\gamma}^{T} \boldsymbol{Y}\left(\sum_{r}^{R} \eta_{r} \boldsymbol{k}_{r}\right) \boldsymbol{\mu} \boldsymbol{y}+\lambda\|\boldsymbol{\gamma}\|^{2}
$$

Where $\gamma$ is the probability distribution of positive and negative training samples, $\boldsymbol{\eta}$ is the base kernel combination vector, a unitary norm vector. $\boldsymbol{H}^{\boldsymbol{t}}$ is the label vector of training set, and $\boldsymbol{k}_{r}$, the $r$-th base kernel. Define the vector of the $r$-th entry $\boldsymbol{d}_{r}(\boldsymbol{\gamma})=\boldsymbol{\gamma}^{T} \hat{\boldsymbol{Y}} \hat{\boldsymbol{K}}_{r} \hat{\boldsymbol{Y}}_{\boldsymbol{\gamma}}$, and then the optimization problem can be written as:

$$
\min _{\gamma \in \Gamma} \max _{\|\eta\|=1}(1-\lambda) \boldsymbol{\eta}^{T} \boldsymbol{d}(\boldsymbol{\gamma})+\lambda\|\boldsymbol{\gamma}\|_{2}^{2}
$$

By maximizing the distance between positive and negative samples, the min-max problem can be simplified as a quadratic problem, of which the analytical solution is as follows:

$$
\boldsymbol{\eta}^{*}=\frac{\boldsymbol{d}\left(\boldsymbol{\gamma}^{*}\right)}{\left\|\boldsymbol{d}\left(\boldsymbol{\gamma}^{*}\right)\right\|_{2}}
$$

The weight parameters of each base kernel $\boldsymbol{K}_{r}$ are obtained through the following formula:

$$
\eta_{r}=\boldsymbol{\gamma}^{* T} \boldsymbol{Y}\left(\boldsymbol{k}_{r} / T_{r}\left(\boldsymbol{k}_{r}\right)\right) \boldsymbol{Y}^{*}
$$

\subsection{NLMKL}

NLMKL is a nonlinear polynomial kernel combination method (Cortes et al. 2009):

$$
\boldsymbol{K}=\sum_{k_{1}+\mathrm{L}+k_{r}=d}^{R} \eta_{1}^{k_{1}} \mathrm{~L} \eta_{r}^{k_{r}} \boldsymbol{K}_{1}^{k_{1}} \mathrm{~L} \quad \boldsymbol{K}_{r}^{k_{r}}, \eta_{r} \geq 0
$$

Where $d$ is the degree of the combination of polynomials, $\boldsymbol{K}$ is a positive semidefinite matrix. In order to reduce the complexity, only the case where $d=2$ is taken into consideration, and thus the nonlinear kernel combination can be given the following expression: 


$$
\boldsymbol{K}=\sum_{k=1}^{R} \sum_{l=1}^{R} \eta_{k} \eta_{l} \boldsymbol{K}_{k} \boldsymbol{K}_{l}
$$

Apply (7) to SVM, and then the weight coefficient $\eta_{r}$ of each base kernel is obtained by solving a min-max problem:

$$
\min _{\eta \in \Omega} \max _{a \in \in_{i}^{m}} \sum_{i=1}^{m} \alpha_{i}-\frac{1}{2} \sum_{i=1}^{m} \sum_{j=1}^{m} \alpha_{i} \alpha_{j} y_{i} y_{j} \mathrm{~K}
$$

Where $\Omega$ is a positive, bounded, and convex set. Norm-1 and norm-2 bounded sets constitute the two possible choices for the set $\Omega$, and the norm-2 bounded set is adopted in this paper.

$$
\Omega_{2}=\left\{\boldsymbol{\eta} \mid \boldsymbol{\eta} \geq 0 \wedge\left\|\boldsymbol{\eta}-\boldsymbol{\eta}_{0}\right\|_{2} \leq \Lambda\right\}
$$

In equation (9), $\boldsymbol{\eta}_{0}$ is the bias parameter of the base kernel weight vector $\boldsymbol{\eta}$. In actual implementation, $\boldsymbol{\eta}_{0}$ is initially taken as 0 and $\Lambda$ as 1 . The min-max optimization problem of equation (8) can be solved by a two-step method. This paper proposes a momentum-based gradient descent algorithm (Algorithm 1). In each iteration, firstly, given the fixation of the base kernel weight vector $\boldsymbol{\eta}$, the support vector machine problem is solved to obtain $\alpha$. Then, considering the constraint $\Omega_{2}$, the base kernel weight vector $\boldsymbol{\eta}$ is updated by the gradient descent method with momentum term while the SVM parameters are fixed. Repeat these two steps until convergence. Because the momentum term increases gradually in the gradient direction while decreasing gradually where the gradient direction changes, we can get faster convergence speed and weaker oscillation.

As the focus of this paper is to develop a machine learning model for spatial prediction of flood susceptibility, we have compared the two proposed MKL models with other algorithms, selecting SVM (linear kernel), SVM (RBF kernel), RMKL (Do et al. 2009), and GMKL (Varma et al. 2009) as baseline methods. The setting particulars 
of the four MKL algorithms (EasyMKL, NLMK, RMKL and GMKL), all of which use linear and RBF kernels as the base kernel, will be covered in Section 5.1.

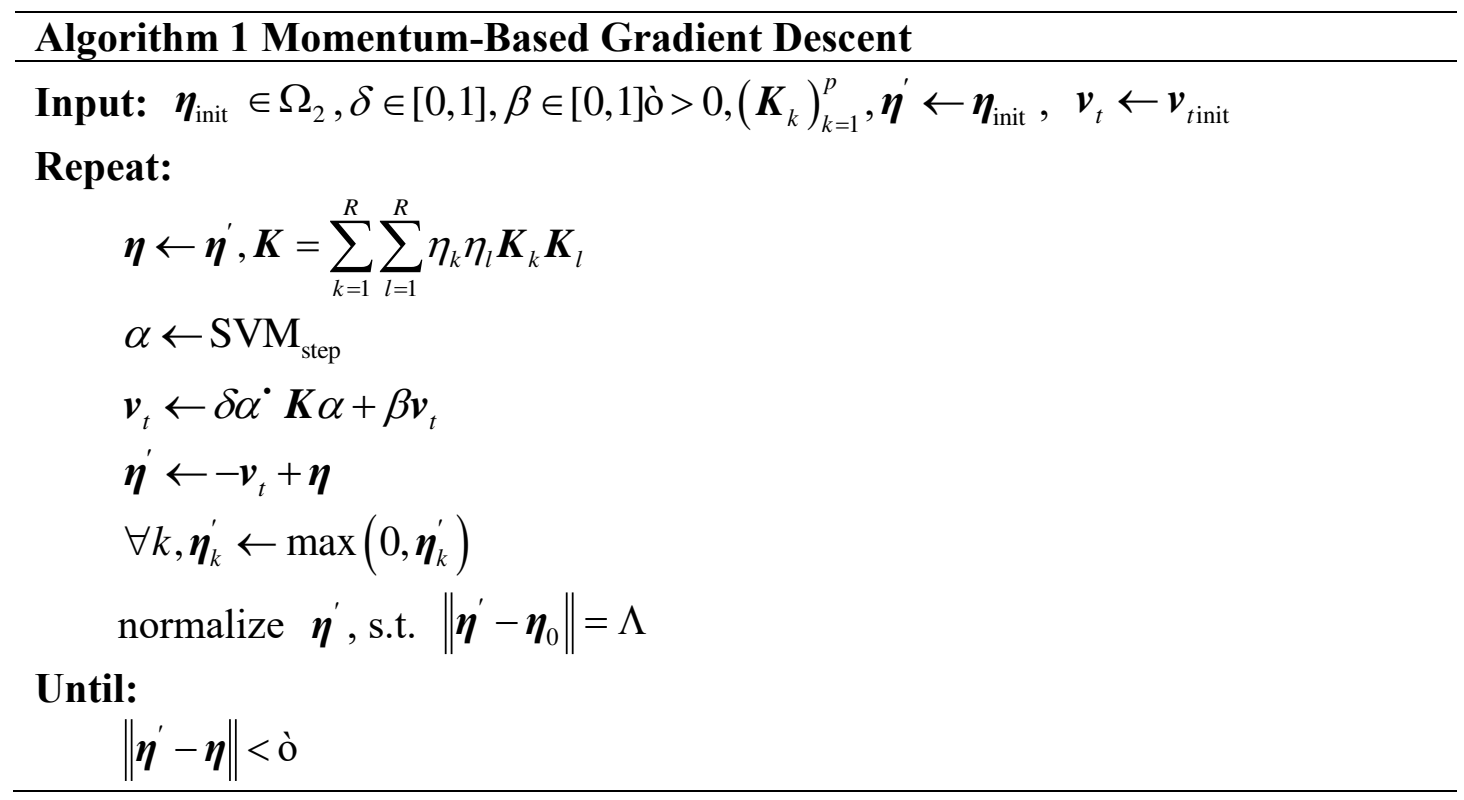

\section{MODIS Imagery Maximum Inundation Extent}

MOD09A1 is a synthetic product of the MODIS surface reflectance by the Terra satellite following an exclusion of interferential factors like atmospheric radiation and aerosol. Its spatial resolution is 500 meters, and its temporal resolution is once every eight days (Chen et al. 2013). MODIS remote sensing imagery is widely used in flood detection and suitable for large-scale spatial research. This study adopts the open water likelihood (OWL) index to extract flood inundation ranges from MOD09A1 remote sensing images (Huang et al. 2014). The OWL index contains five parameters sensitive to water bodies, namely, the short-wave infrared band (band 6 and band 7), NDVI (Townshend et al. 1986), NDWI (Gao et al. 1995) and the multi-resolution valley bottom flatness index (MrVBF) (Gallant et al. 2003). Modeled by nonlinear logistic regression, the OWL index adopts the expression below:

$$
\mathrm{OWL}=\frac{1}{1+\exp (f)}
$$

Where 


$$
f=\mathrm{a}_{0}+\sum_{i=1}^{5} \mathrm{a}_{i} x_{i}
$$

Where $\mathrm{x} 1=$ SWIR Band $6($ reflectance $\times 1000) ; \mathrm{x} 2=$ SWIR Band 7 (reflectance $\times 1000) ; \mathrm{x} 3=\mathrm{NDVI} ; \mathrm{x} 4=\mathrm{NDWI} ; \mathrm{x} 5=$ MrVBF; $\mathrm{a} 0=-3.41375620 ; \mathrm{a} 1=$ $-0.000959735270 ; \mathrm{a} 2=0.00417955330 ; \mathrm{a} 3=14.1927990 ; \mathrm{a} 4=-0.430407140 ; \mathrm{a} 5=$ -0.0961932990 . The OWL index value ranges from 0 to 100 , representing the probability of water existence in each pixel of the MOD09A1 remote sensing images. Whether the pixel is a flooded or non-flooded one is determined by setting the corresponding threshold. In this study, the threshold is set as 0 , so if the value exceeds 0 , it is judged to be a flooded pixel.

The flood inundation range of a single image is obtained through the OWL index. ArcGIS can help synthesize multiple flood inundation ranges of the same study area throughout a long time series into the maximum flood inundation range, which is used as the base map for the sampling of the machine learning model. The chosen sample sites are labeled "flooded" or "non-flooded".

\section{Data Description}

\subsection{Study area and historical daily flow data}

This paper examines the area between Sanmenxia and Huayuanko (A.K.A. the Sanhuajian Basin) in the middle reaches of the Yellow River, the longitude between $109.73^{\circ} \mathrm{E}$ and $113.58^{\circ} \mathrm{E}$, and the latitude between $33.64^{\circ} \mathrm{N}$ and $36.97^{\circ} \mathrm{N}$. Sanhuajian is surrounded by mountains in the north, west and south; the Yellow River runs from west to east across it in the middle. A semi-humid area, Sanhuajian witnesses frequent rainstorms from late June to early August (Wang et al. 2014).

We obtained the historical daily flow data of the Huayuankou Site from the YRCC 
website $^{1}$, which cover ten complete water years (from 2007 to 2017) as shown in Fig. 1 Flooding is usually caused by flood peaks, and the major peaks are represented by red dots in the Fig. 1 .

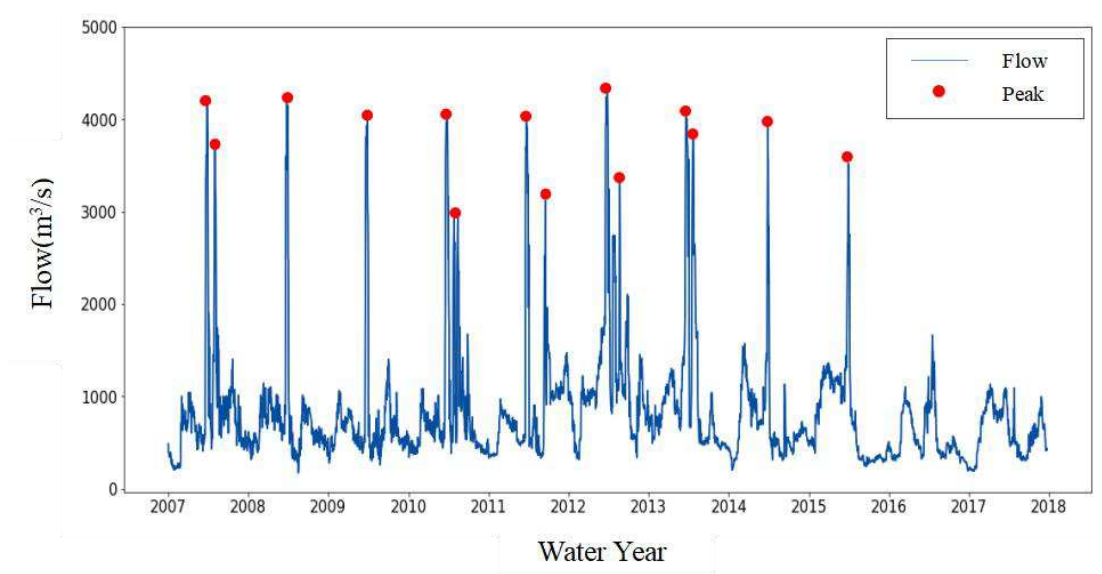

Fig. 1 Historical data of the daily flow of the Huayuankou Site.

\subsection{Flood inventories}

According to the time points of flood peak occurrences in Fig. 1, we pinpointed the corresponding dates of the MOD09A1 remote sensing images, along with the dates before and after the occurrences. The flood inundation range of each remote sensing image was extracted by OWL index and synthesized via ArcGIS into the maximum flood inundation range of the study area.

In the maximum flood inundation range, 310 flooded sites were randomly selected; in the non-flood inundation range, 310 non-flooded sites were randomly selected. In Fig. 2, the red dots stand for flooded sites, and the blue dots, non-flooded sites. We used $70 \%$ (434) of the total sample sites as the training set and $30 \%(186)$ as the test set, marking the flooded sites as " 1 " and the non-flooded sites as "-1".

\footnotetext{
${ }^{1}$ http://www.yrcc.gov.cn/
} 


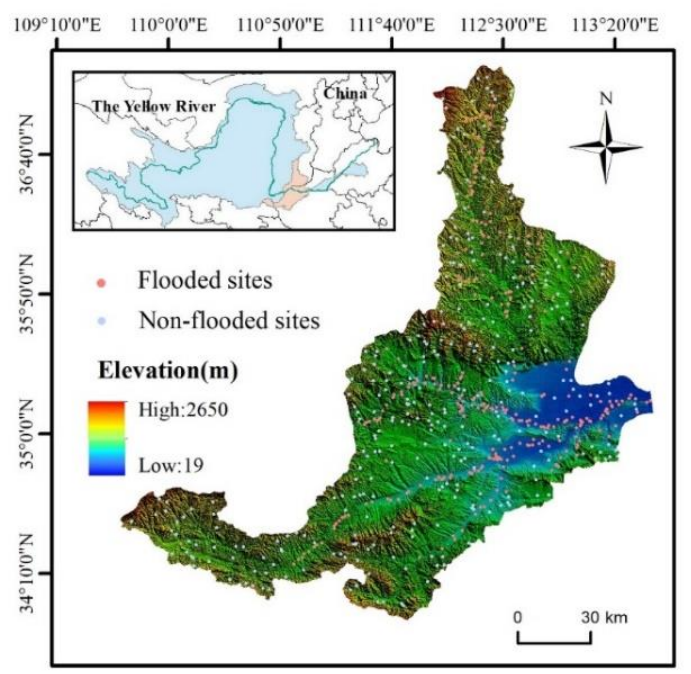

Fig. 2 Location of flooded and non-flooded sites in Sanhuajian.

\subsection{Flood conditioning factors}

Flood disasters involve an extremely complex process and can be attributed to many interplaying factors. Drawing reference from previous studies (Zhao et al. 2019; Dodangeh et al. 2020; Tien et al. 2020), this paper selects out ten flood conditioning factors, including elevation, slope, aspect, curvature, Stream Power Index (SPI), Topographic Wetness Index (TWI), distance from river, soil type, vegetation, and annual average rainfall. Table 1 lists the data source, format, spatial and temporal resolution of these flood conditioning factors. Stream Power Index (SPI) represents the river erosivity of the catchment area, while Topographic Wetness Index (TWI) takes into account the influence of the terrain and soil characteristics on soil moisture distribution (Regmi et al. 2010). They are calculated using the following formulas:

$$
\begin{gathered}
\mathrm{SPI}=A \tan \beta / b \\
\mathrm{TWI}=\ln (A / b \tan \beta)
\end{gathered}
$$

Where $A\left(\mathrm{~m}^{2}\right)$ is the upstream catchment area or flow accumulation, $b(\mathrm{~m})$ is the unit width of water flow, and $\beta$ (radian) is the slope.

Because the resolution of each factor is different, we used ArcGIS to preprocess 
all the data and resampled them into raster data with a resolution of $90 \mathrm{~m}$, so that all raster data share the same coordinate system and pixel size, making it convenient for machine learning and statistical analysis. Flood conditioning factors are shown in Fig.

3.

Table 1 Data source of flood conditioning factors.

\begin{tabular}{|c|c|c|c|c|}
\hline name & $\begin{array}{l}\text { data } \\
\text { format }\end{array}$ & $\begin{array}{c}\text { spatial } \\
\text { resolution }\end{array}$ & $\begin{array}{l}\text { temporal } \\
\text { resolution }\end{array}$ & source \\
\hline DEM & TIF & $30 \mathrm{~m}$ & 2015 & ASTER GDEM V2 \\
\hline slope & TIF & $30 \mathrm{~m}$ & 2015 & DEM \\
\hline aspect & TIF & $30 \mathrm{~m}$ & 2015 & DEM \\
\hline curvature & TIF & $30 \mathrm{~m}$ & 2015 & DEM \\
\hline SPI & TIF & $30 \mathrm{~m}$ & & DEM \\
\hline TWI & TIF & $30 \mathrm{~m}$ & & DEM \\
\hline $\begin{array}{l}\text { Distance } \\
\text { from river }\end{array}$ & shapefile & & & DEM \\
\hline Soil types & shapefile & $1: 1000000$ & 1995 & $\begin{array}{c}\text { CASEarth Data Sharing and } \\
\text { Service } \text { Portal }^{2}\end{array}$ \\
\hline vegetation & shapefile & $1: 1000000$ & 2007 & $\begin{array}{c}\text { CASEarth Data Sharing and } \\
\text { Service Portal }\end{array}$ \\
\hline rainfall & TIF & $\begin{array}{c}0.5^{\circ} \times \\
0.5^{\circ}\end{array}$ & $\begin{array}{l}2007- \\
2017\end{array}$ & $\begin{array}{c}0.5^{\circ} \times 0.5^{\circ} \text { raster data set of } \\
\text { monthly surface precipitation in } \\
\text { China }(\mathrm{V} 2.0)^{3}\end{array}$ \\
\hline 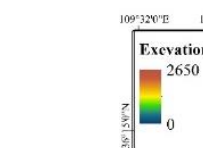 & & wert & & 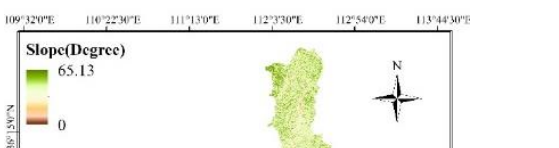 \\
\hline
\end{tabular}

${ }^{2}$ http://data.casearth.cn/

${ }^{3}$ http://data.cma.cn/data/cdcdetail/dataCode/SURF_CLI_CHN_PRE_MON_GRID_0.5.html 

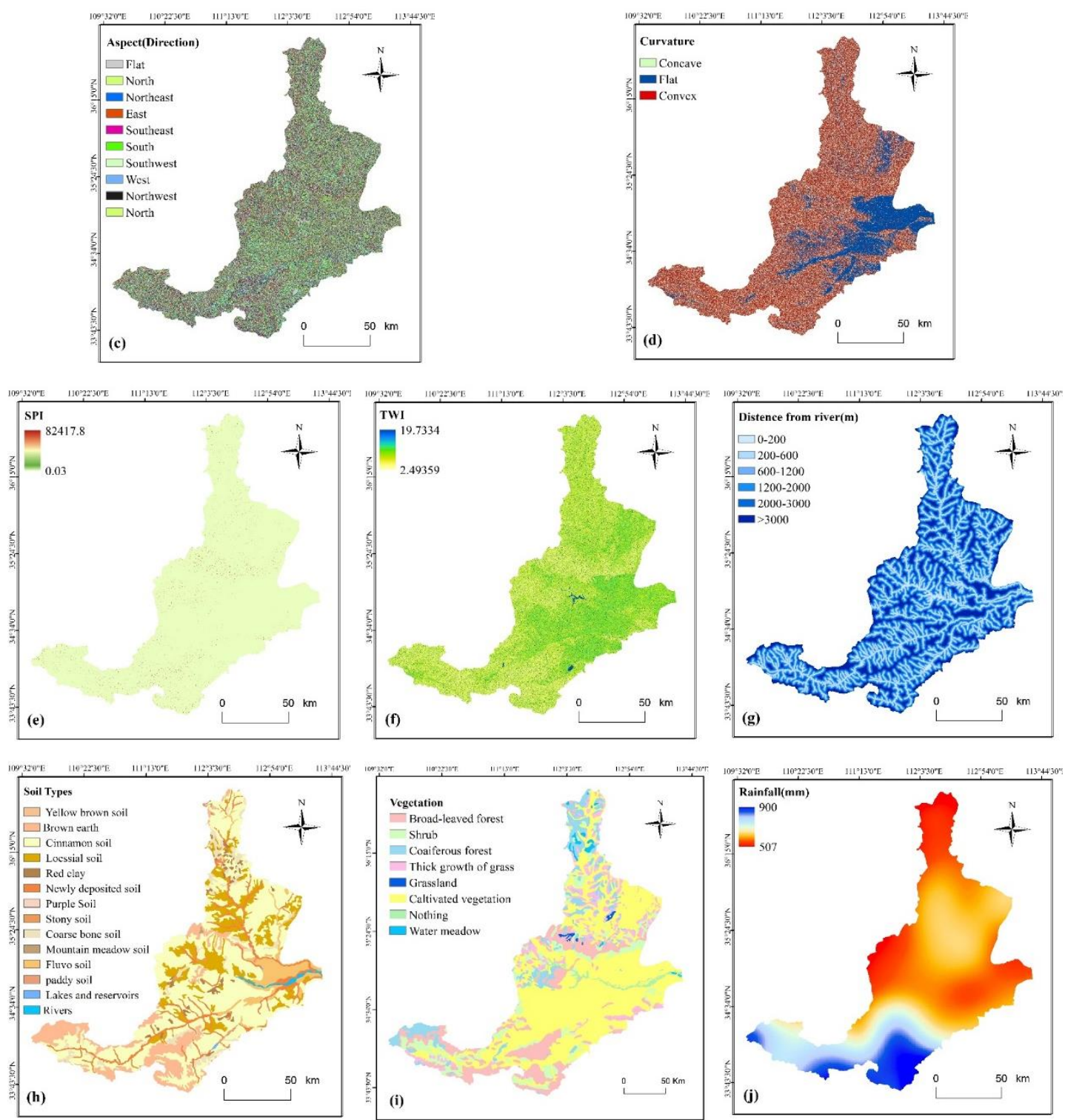

Fig. 3 Flood conditioning factors:(a) Elevation (meter), (b) Slope (degree), (c) Aspect, (d) Curvature, (e) SPI, (f) TWI, (g) Distance to rivers (meter), (h) Soil Types, (i) Vegetation, (j) Rainfall (mm).

\section{Experimental Results and Discussions}

\subsection{Experiment settings}

The historical flood inundation data set includes 620 instances (positive and negative instances are equal in number), among which 434 are used for training (70\%) and 186 for testing. The dimension (feature length) of each instance is 10 . In order to get the best performance, the data set has been standardized. 
SVM (linear kernel), SVM (RBF kernel), RMKL and GMKL were adopted as the baseline methods. EasyMKL, NLMK, RMKL and GMKL all used linear and RBF kernels as the base kernels.

We implemented these algorithms in Python using Sklearn Library and PyTorch Library and found the best hyper parameter configuration through 5-fold crossvalidation and grid search: $\mathrm{C}$ for SVM and $\gamma$ for RBF kernel function. The value range of $C$ is $C=\left\{10^{-2}, 10^{-1}, \ldots, 10^{2}\right\}$, and that of $\gamma$ is $\gamma=\{0.01,0.1,0.2,0.5,0.8,1\}$. Accuracy is the evaluation criterion of the cross-validation.

\subsection{Performance evaluation}

The performance of each model was evaluated in terms of accuracy (ACC), F1 score, and Area Under Curve (AUC) - common indicators for machine learning model evaluation (Choubin et al. 2019; Hu et al. 2021). TP, TN, FP, and FN were true positive (correctly classified flooded sites), true negative (correctly classified non-flooded sites), false positive (misclassified flooded sites) and false negative (misclassified nonflooded sites), respectively.

$$
\begin{array}{r}
\mathrm{ACC}=\frac{T P+T N}{T P+F P+T N+F N} \\
\mathrm{~F} 1=\frac{2 \times P \times R}{P+R}
\end{array}
$$

$P$ and $R$ are precision and recall:

$$
\begin{aligned}
& P=\frac{T P}{T P+F P} \\
& R=\frac{T P}{T P+F N}
\end{aligned}
$$

As to the receiver operating characteristic (ROC) curve, false positive rate (FPR) is defined as the $\mathrm{X}$-axis, and true positive rate (TPR), the Y-axis. Area Under Curve 
(AUC), which is widely applied in FSM model evaluation, was used to assess the model performance. When AUC is 0.5 , it means the model works like a random model; when AUC exceeds 0.8, it shows the model has good performance (Tien et al. 2016); when AUC is 1 , the model features perfect classification.

\subsection{Experiment results and analysis}

Table 2 presents a detailed comparison of the classification algorithms used in the training set. The performance of the multiple kernel method is better than that of the single kernel support vector machine. In terms of accuracy, NLMKL is the best (0.938), followed by EasyMKL (0.917) and GMKL(0.912). In terms of F score, NLMKL (0.941) and EasyMKL (0.92) are the best performers. Fig. 4 shows the ROC curve and AUC value of each model in the training set. EasyMKL (0.973) and NLMKL (0.987) have higher AUC values, similar ROC curves, and therefore similar performances, followed by GMKL(0.970). Table 3 presents a detailed comparison of the classification algorithms used in the test set. In general, the multiple kernel method again outperforms the single kernel support vector machine. In terms of accuracy, NLMKL is the best (0.833), followed by EasyMKL (0.823) and GMKL (0.817). With regard to the F score, the ranking remains unchanged. Fig. 5 shows the ROC curve and AUC value of each model in the training set. All classification algorithms show similar performance, yet NLMKL (0.889) has a higher AUC value.

Table 2 Performance of different models in training set.

\begin{tabular}{lllllll}
\hline & SVM(linear) & SVM(RBF) & EasyMKL & NLMKL & RMKL & GMKL \\
\hline ACC & 0.800 & 0.876 & 0.917 & 0.938 & 0.901 & 0.912 \\
F & 0.811 & 0.884 & 0.92 & 0.941 & 0.904 & 0.916 \\
P & 0.786 & 0.851 & 0.908 & 0.919 & 0.878 & 0.9 \\
R & 0.838 & 0.919 & 0.933 & 0.964 & 0.933 & 0.933 \\
\hline
\end{tabular}


Table 3 Performance of different models in test set.

\begin{tabular}{lllllll}
\hline & SVM(linear) & SVM(RBF) & EasyMKL & NLMKL & RMKL & GMKL \\
\hline ACC & 0.806 & 0.801 & 0.823 & 0.833 & 0.812 & 0.817 \\
F & 0.816 & 0.810 & 0.833 & 0.841 & 0.821 & 0.821 \\
P & 0.734 & 0.731 & 0.762 & 0.759 & 0.741 & 0.757 \\
R & 0.919 & 0.908 & 0.92 & 0.943 & 0.919 & 0.897 \\
\hline
\end{tabular}

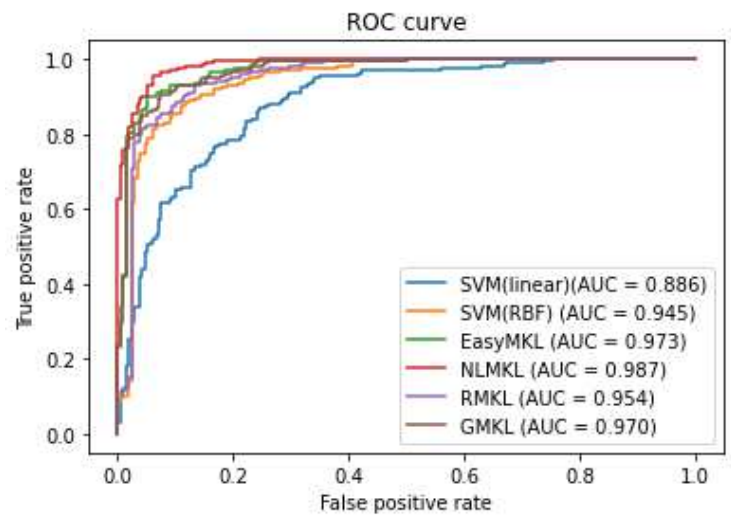

Fig. 4 ROC and AUC of different models in training set.

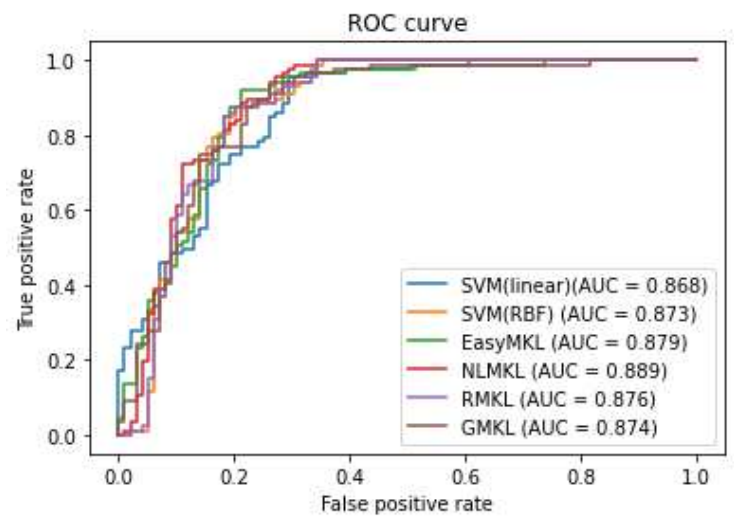

Fig. 5 ROC and AUC of different models in test set.

The trained machine learning model was used to predict the probability of flood inundation for each pixel in the study area. The classification methods commonly used in GIS include natural discontinuity classification method, quantile method, equal interval method, and geometric interval method (Tehrany et al. 2013). The natural discontinuity method, based on the inherent natural grouping of data, can identify the similar values and group them in the most appropriate manner (Smith et al. 2007). It 
can also maximize the difference between classes and set the boundary at the point where the difference of data values is relatively large. Therefore, we used the natural discontinuity classification method in ArcGIS to divide the model-obtained probability values into five categories (very low, low, moderate, high, and very high) and completed the flood susceptibility map of the study area (Fig. 6). As can be seen from all the figures, the most flood-vulnerable areas are mainly located in the east of the basin. These areas are low and flat, and placed in proximity of the river and most of the vegetation there is cultivated.
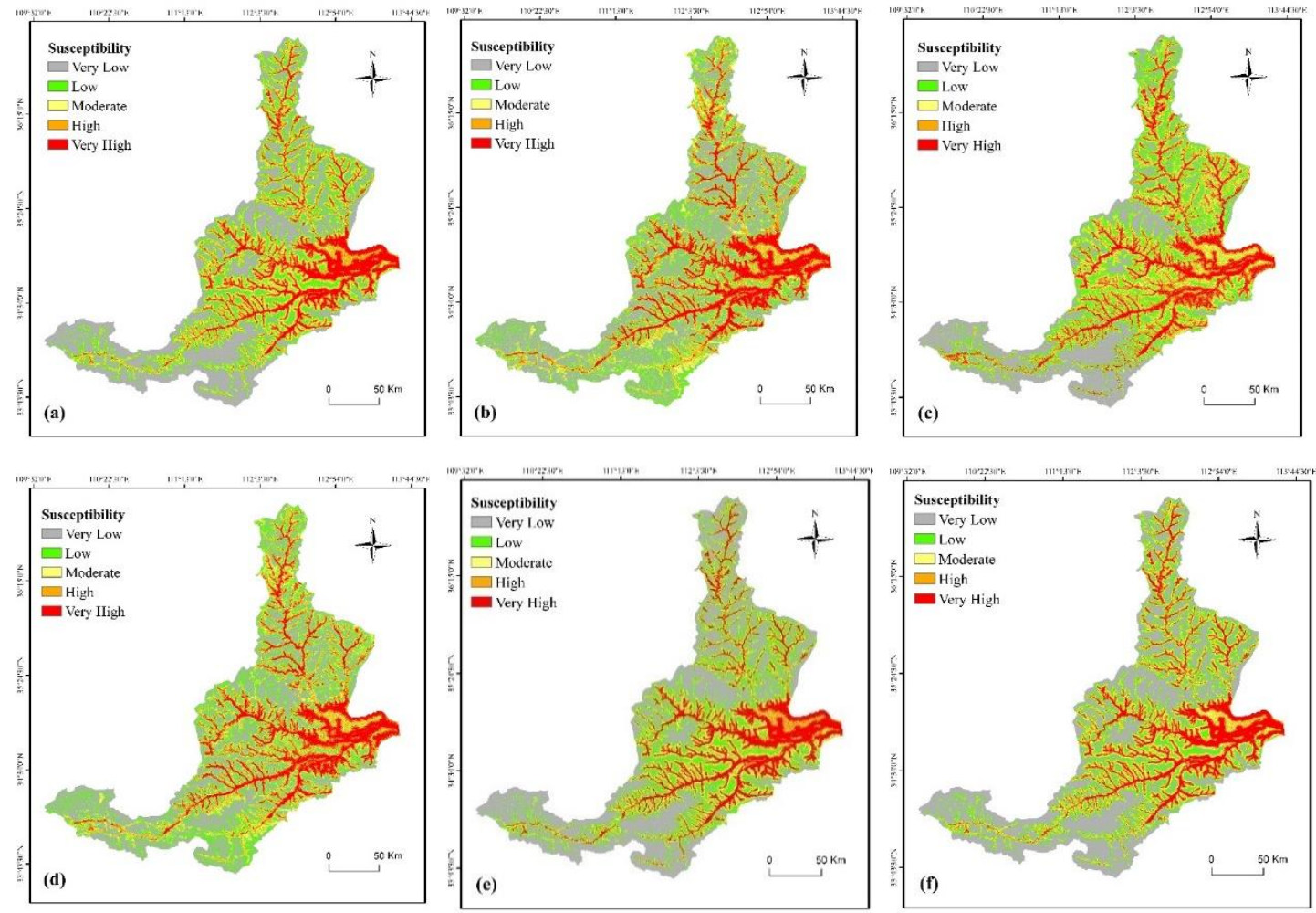

Fig. 6 Flood susceptibility maps derived from (a) SVM (linear), (b) SVM (RBF), (c) EasyMKL, (d) NLMKL, (e) RMKL, (f) GMKL.

Table 4 Flood density on flood susceptibility maps by the five models

\begin{tabular}{ccccccc}
\hline & Flood & Number & Nols of \\
Model & $\begin{array}{c}\text { susceptibility } \\
\text { level }\end{array}$ & $\begin{array}{c}\text { Pixels } \\
\text { class }\end{array}$ & \%class & $\begin{array}{c}\text { of } \\
\text { flooded } \\
\text { sites }\end{array}$ & $\begin{array}{c}\text { flooded } \\
\text { sites }\end{array}$ & $\begin{array}{c}\text { Flood } \\
\text { density }\left(1 / 10^{6}\right)\end{array}$ \\
\hline SVM(linear) & Very low & 1484188 & 33.09 & 5 & 1.61 & 3.37
\end{tabular}




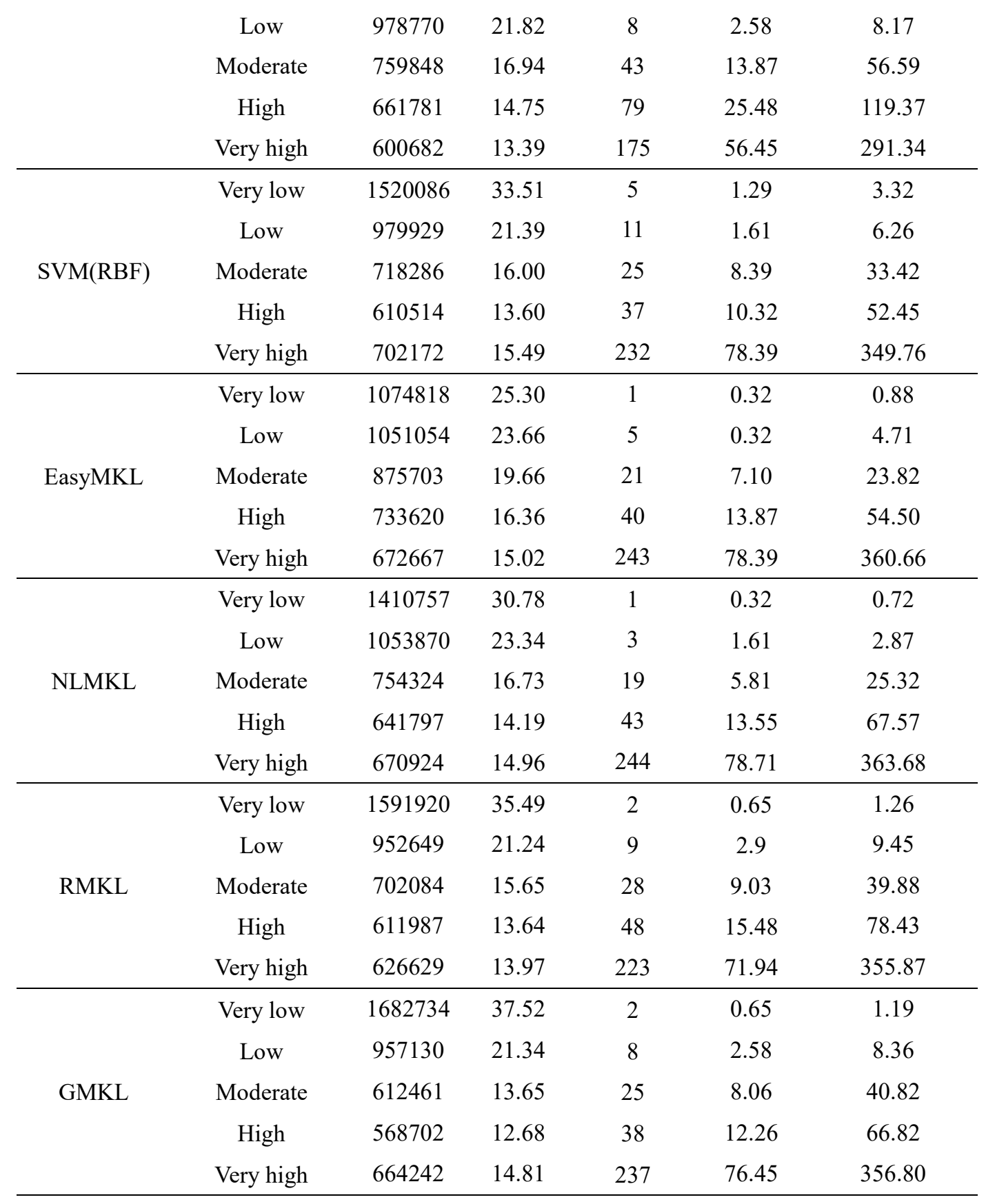




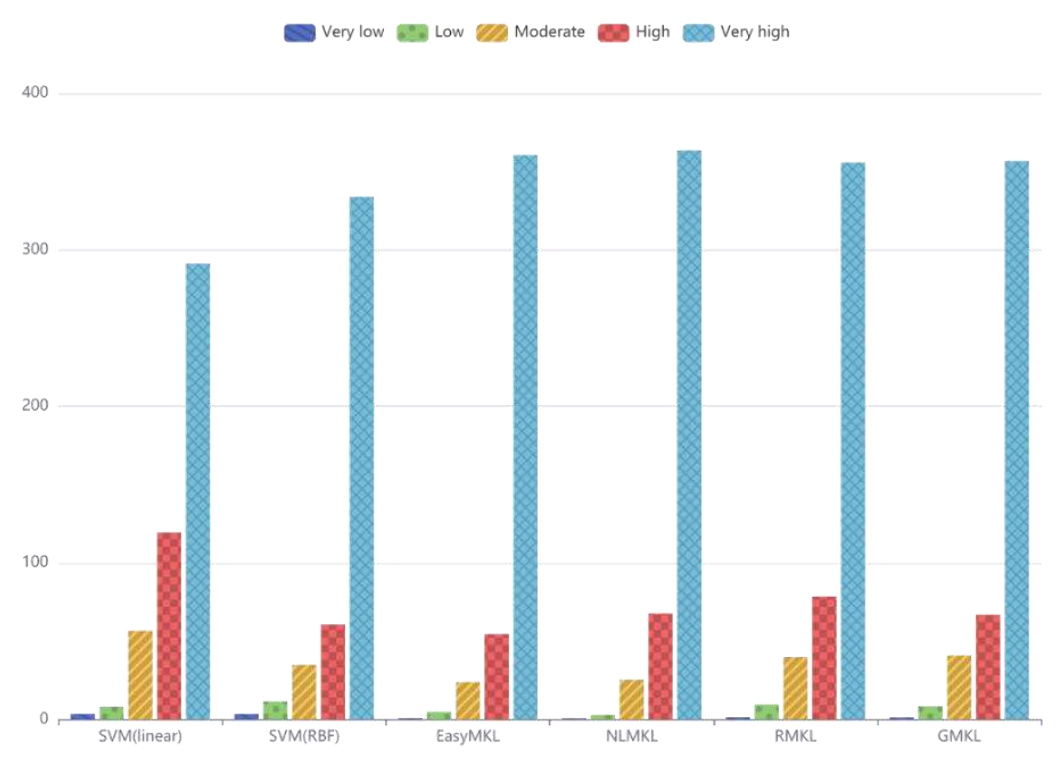

Fig. 7 Flood density in different flood susceptibility areas by the six models.

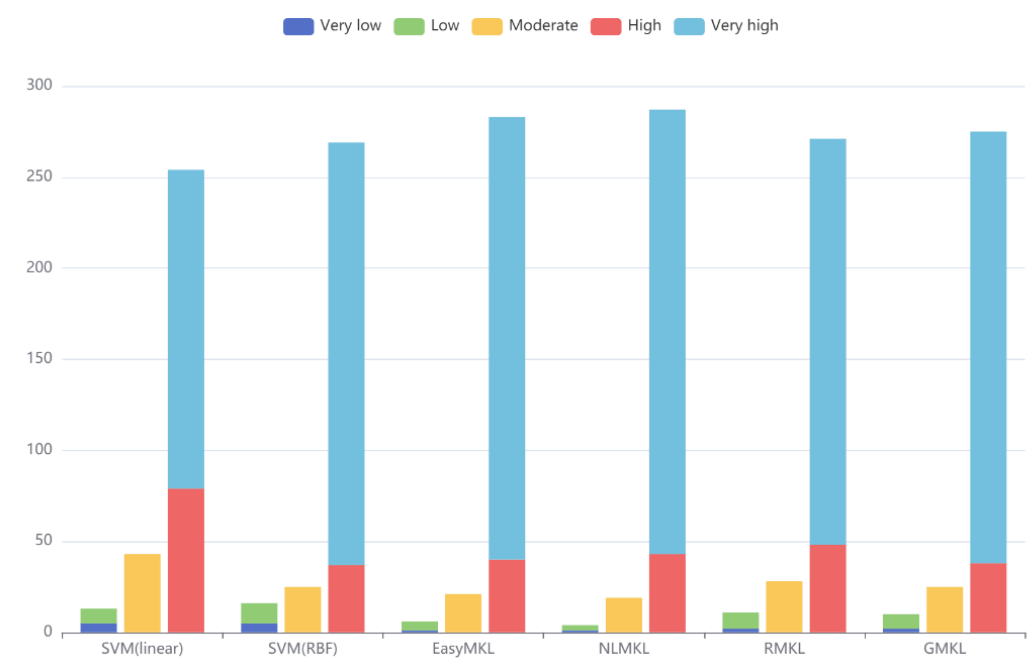

Fig. 8 Flooded sites in different flood susceptibility areas by the six models.

Flood density was taken into consideration to further evaluate the performance of the flood susceptibility map (Chapi et al. 2017). FD is the ratio of flooded site number to class pixel number in each susceptibility category. Ideally, density values should increase from very low susceptibility areas to very high susceptibility areas (Pradhan et al. 2010). Table 4 lists the distribution of the categories of flood susceptibility maps by each model, the number of flood sites in each category, and flood density. Fig. 7 shows flood density in different flood susceptibility areas by the six compared models. The results show that the flood susceptibility map obtained by the NLMKL (363.68) model 
has the highest FD in extremely high susceptibility area. Also a multiple kernel learning method, EasyMKL (360.66) shows great performance, which is superior to other baseline methods. Likewise, in very low and low susceptibility areas, the multiple kernel learning method outperforms the single kernel SVM. Among those in comparison, NLMKL (0.72 and 2.87) is the best performer. In terms of flood site coverage, Fig. 8 shows the inundation sites in different flood susceptibility areas by the six compared models. EasyMKL and NLMKL cover 283 and 287 flood sites respectively in the high and very high susceptibility areas, outperforming the linear kernel SVM (254), RBF kernel SVM (269), RMKL (271), and GMKL (275); in low and very low susceptibility areas, EasyMKL and NLMKL cover six and four flood sites respectively, outperforming the linear kernel SVM (11), RBF kernel SVM (16), RMKL (11), and GMKL (10) .

\section{Conclusion}

In this study, two multiple kernel learning methods were developed for flood susceptibility mapping of Sanhuajian of the Yellow River. The performance of the two MKL methods (EasyMKL and NLMKL) is compared with that of the linear kernel SVM, RBF kernel SVM, RMKL, and GMKL. The results show that the MKL method has better performance and is more suitable for flood susceptibility mapping, especially for flood inundation sites extracted from historical MODIS remote sensing images alone. To summarize, the major contributions of this study are as follows:

(1) In the absence of any historical records of the inundation sites, the sites are pinpointed using MODIS remote sensing images to provide data sets for machine learning models and complete the flood susceptibility map of the study area. This data acquisition method can provide reference to other areas in need of a flood susceptibility map when lacking a record of the inundation sites. 
(2) The two MKL models have higher prediction accuracy and higher quality in generating the flood susceptibility map. Their performance is better than that of the single kernel support vector machine.

(3) Between the two MKL methods, NLMKL has the higher prediction accuracy, F1 score, and AUC than EasyMKL. In terms of the flood susceptibility map performance, NLMKL is also slightly better than EasyMKL.

Authors Contributions Q. Hu: Conceptualization, Formal analysis, Investigation, Methodology, Software, Validation, Roles/Writing - original draft. Y.L. Zhu: Funding acquisition, Project administration, Writing - review \& editing. H.X. Hu: Conceptualization, Methodology, Supervision, Writing - review \& editing. Z. Guan: Data curation, Software, Visualization. Z.Y. Qian: Data curation, Software, Visualization. A. Yang: Resources, Project administration.

Funding This work was supported by National Key Research and Development Program of China (Grant No: 2018YFC0407904).

\section{Declarations}

Ethical Approval All authors have seen and agreed with the contents of the manuscript and are looking forward to publishing this paper on "Water Resources Management" journal.

Consent to Participate Not applicable.

Consent to Publish Not applicable.

Conflict of Interest The authors declare that there is no conflict of interest.

Competing Interests The authors declare no competing interests. 


\section{Reference}

Aiolli F, Donini M (2015) EasyMKL: a scalable multiple kernel learning algorithm. Neurocomputing 169:215-224. https://doi.org/10.1016/j.neucom.2014.11.078

Arratia A, Belanche L A, Fábregues L (2020) An Evaluation of Equity Premium Prediction Using Multiple Kernel Learning with Financial Features. Neural Process Lett 52(1):117-134. https://doi.org/10.1007/s11063-018-09971-7

Beilicci E, Beilicci R, Visescu M (2016) Study of flooding risk areas using 1D/2D advanced hydroinformatic tools. The 16th International Multidisciplinary Scientific GeoConference-SGEM

Chapi K, Singh V P, Shirzadi A, Shahabi H, Bui D T, Pham B T, Khosravi K (2017) A novel hybrid artificial intelligence approach for flood susceptibility assessment. Modell Softw 95:229-245. http://doi.org/10.1016/i.envsoft.2017.06.012

Chen B, Shi F, Lin T, Shi P, Zheng J (2020) Intensive Versus Extensive Events? Insights from Cumulative Flood-Induced Mortality Over the Globe, 1976-2016. Int J Disaster Risk Sci 11:441-451. https://doi.org/10.1007/s13753-020-00288-5

Chen Y, Huang C, Ticehurst C, Merrin L, Thew P (2013) An Evaluation of MODIS Daily and 8-day Composite Products for Floodplain and Wetland Inundation Mapping. Wetlands 33(5):823-835. https://doi.org/10.1007/s13157-013-0439-4

Choubin B, Moradi E, Golshan M, Adamowski J, Sajedi-Hosseini F, Mosavi A (2019) An ensemble prediction of flood susceptibility using multivariate discriminant analysis, classification and regression trees, and support vector machines. Sci Total 
Environ 651:2087-2096. https://doi.org/10.1016/j.scitotenv.2018.10.064

Cortes C, Mohri M, Rostamizadeh A (2009) Learning Non-Linear Combinations of Kernels. Advances in Neural Information Processing Systems, pp 396-404

Damasevicius R (2010) Optimization of SVM parameters for recognition of regulatory DNA sequences. TOP 18(2):339-353. https://doi.org/10.1007/s11750-010-0152-x

Dodangeh E, Choubin B, Eigdir A N, Nabipour N, Panahi M, Shamshirband S, Mosavi A (2020) Integrated machine learning methods with resampling algorithms for flood susceptibility prediction. Sci Total Environ 705:135983. https://doi.org/10.1016/j.scitotenv.2019.135983

Do H, Kalousis A, Woznica A, Hilario M (2009) Margin and Radius Based Multiple Kernel Learning. Machine Learning and Knowledge Discovery in Databases, Springer, Berlin, Heidelberg, pp 330-343

Gao B C (1995) NDWI - A Normalized Difference Water Index for remote sensing of vegetation liquid water from space. Remote Sensing of Environment. Remote Sens. Environ 58:257-266. https://doi.org/10.1117/12.210877

Gallant J C, Dowling T I (2003) A multiresolution index of valley bottom flatness for mapping depositional areas. Water Resour. Res 39(12):1347-1359. https://doi.org/10.1029/2002WR001426

Gönen M, Alpaydin E (2011) Multiple Kernel Learning Algorithms. J Mach Learn Res 12:2211-2268. https://dl.acm.org/doi/10.5555/1953048.2021071

Gönen M, Alpaydın E (2013) Localized algorithms for multiple kernel learning. Pattern 
Recognit 46(3):795-807. https://doi.org/10.1016/j.patcog.2012.09.002

Gu Y, Liu T, Jia X, Benediktsson J A, Chanussot J (2016) Nonlinear Multiple Kernel Learning With Multiple-Structure-Element Extended Morphological Profiles for Hyperspectral Image Classification. IEEE Trans Geosci Remote Sensing 54(6):3235-3247. http://doi.org/10.1109/TGRS.2015.2514161

Hoch J M, van Beek R, Winsemius H C, Bierkens M F P (2018) Benchmarking flexible meshes and regular grids for large-scale fluvial inundation modelling. Adv Water Resour 121:350-360. https://doi.org/10.1016/j.advwatres.2018.09.003

Huang C, Chen Y, Wu J (2014) Mapping spatio-temporal flood inundation dynamics at large river basin scale using time-series flow data and MODIS imagery. Int J Appl Earth Obs Geoinf 26:350-362. http://doi.org/10.1016/j.jag.2013.09.002

Hu H, Li Q, Zhao Y, Zhang Y (2021) Parallel Deep Learning Algorithms With Hybrid Attention Mechanism for Image Segmentation of Lung Tumors. IEEE Trans Ind Inform 17(4):2880-2889. https://doi.org/10.1109/TII.2020.3022912

Mateo C M R, Yamazaki D, Kim H, Champathong A, Vaze J, Oki T (2017) Impacts of spatial resolution and representation of flow connectivity on large-scale simulation of floods. Hydrol Earth Syst Sci 21:5143-5163. https://doi.org/10.5194/hess-21-

\section{$\underline{5143-2017}$}

Pappenberger F, Beven K, Horritt M, Blazkova S (2005) Uncertainty in the calibration of effective roughness parameters in HEC-RAS using inundation and downstream level observations. J Hydrol 302(1-4):46-69. 


\section{https://doi.org/10.1016/j.jhydrol.2004.06.036}

Pradhan B, Lee S (2010) Landslide susceptibility assessment and factor effect analysis: backpropagation artificial neural networks and their comparison with frequency ratio and bivariate logistic regression modelling. Modell Softw 25(6):747-759. http://doi.org/10.1016/j.envsoft.2009.10.016

Qi R, Wu J, Guo F, Xu L, Zou Q (2020) A spectral clustering with self-weighted multiple kernel learning method for single-cell RNA-seq data. Brief Bioinform bbaa216. https://doi.org/10.1093/bib/bbaa216

Rajib A, Liu Z, Merwade V, Tavakoly A, Follum M (2020) Towards a large-scale locally relevant flood inundation modeling framework using SWAT and LISFLOOD-FP. J Hydrol 581:124406. https://doi.org/10.1016/j.jhydrol.2019.124406

Rakotomamonjy A, Bach F R, Canu S, Grandvalet Y (2008) SimpleMKL. J Mach Learn Res 9: 2491-2521

Regmi N R, Giardino J R, Vitek J D (2010) Modeling susceptibility to landslides using the weight of evidence approach: Western Colorado, USA, Geomorphology 115(12):172-187. https://doi.org/10.1016/j.geomorph.2009.10.002

Shafapour T M, Kumar L, Neamah J M, Shabani F (2019) Evaluating the application of the statistical index method in flood susceptibility mapping and its comparison with frequency ratio and logistic regression methods. Geomatics Nat Hazards Risk 10(1):79-101. https://doi.org/10.1080/19475705.2018.1506509

Smith M J, Goodchild M F, Longley P A (2007) Geospatial Analysis: A Comprehensive 
Guide to Principles, Techniques and Software Tools (2. Ed.). DBLP

Tehrany M S, Pradhan B, Jebur M N (2013) Spatial prediction of flood susceptible areas using rule based decision tree (DT) and a novel ensemble bivariate and multivariate statistical models in GIS. J Hydrol 504:69-79. http://doi.org/10.1016/j.jhydrol.2013.09.034

Teng J, Jakeman A J, Vaze J, Croke B F W, Dutta D, Kim, S (2017) Flood inundation modelling: A review of methods, recent advances and uncertainty analysis. Environ Modell Softw. 90:201-216. http://doi.org/10.1016/j.envsoft.2017.01.006

Tien B D, Hoang N, Martínez-álvarez F, Ngo P T, Hoa P V, Pham T D, Samui P, Costache R (2020) A novel deep learning neural network approach for predicting flash flood susceptibility: A case study at a high frequency tropical storm area. Sci Total Environ 701:134413. https://doi.org/10.1016/j.scitotenv.2019.134413

Tien B D, Tuan T A, Klempe H, Pradhan B, Revhaug I (2016) Spatial prediction models for shallow landslide hazards: a comparative assessment of the efficacy of support vector machines, artificial neural networks, kernel logistic regression, and logistic model tree. Landslides 13(2):361-378. https://doi.org/10.1007/s10346-015-0557$\underline{6}$

Townshend J R G, Justice C O (1986) Analysis of the dynamics of African vegetation using the normalized difference vegetation index. Int J Remote Sens 7:1435-1445. https://doi.org/10.1080/01431168608948946

Varma M, Babu B (2009) More generality in efficient multiple kernel learning, 
International Conference on Machine Learning, ACM, pp 1065-1072

Wang Z, Zheng S (2014) Application of the Meteorological and Hydrological Models Coupling in the Flood Forecasting in Sanmenxia-Huayuankou Reach of the Yellow River. Meteorological and Environmental Sciences 37(02):8-13 (In Chinese)

Yariyan P, Janizadeh S, Van Phong T, Nguyen H D, Costache R, Van Le H, Pham B T, Pradhan B, Tiefenbacher J P (2020) Improvement of Best First Decision Trees Using Bagging and Dagging Ensembles for Flood Probability Mapping. Water Resour Manag 34(9):3037-3053. https://doi.org/10.1007/s11269-020-02603-7

Zhao G, Pang B, Xu Z, Peng D, Xu L (2019) Assessment of urban flood susceptibility using semi-supervised machine learning model. Sci Total Environ 659: 940-949. https://doi.org/10.1016/i.scitotenv.2018.12.217

Zheng H, Wang R, Yu Z, Wang N, Gu Z, Zheng B (2017) Automatic plankton image classification combining multiple view features via multiple kernel learning. BMC Bioinformatics 18(S16):518-570. https://doi.org/10.1186/s12859-017-1954-8 


\section{Supplementary Files}

This is a list of supplementary files associated with this preprint. Click to download.

- graphicalabstract.docx 